\title{
Hearing Outcomes of Cochlear Implant Recipients With Preoperatively Identified Cochlear Dead Regions
}

Lee JW, Shrivastava M, Bird J, Tysome JR, Donnelly NP, Axon PR, Bance ML

Otology and Skull Base Unit/Emmeline Centre, Cambridge University Hospitals

\section{Introduction}

- There are 16200 cochlear implant patients in the UK, with 1400 new CI recipients in 2016-17 (British Cochlear Implant Group)

- Vast majority of patients receiving CIs fulfil the traditional NICE guidance criteria

- In our institution, we offer CIs to patients who do not fulfil traditional criteria, but are identified as having cochlear dead regions

- Cochlear dead regions (DRs)

Absence of function of inner hair cells or auditory neurons at the

characteristic frequency (CF)

- Contribute to poor auditory perception

- Associated with worse outcomes with conventional hearing aids

- Sound stimulus falling within a DR may register a threshold on pure tone audiogram (PTA) due to off frequency listening

Patients do worse on functional testing than expected from PTA

- Threshold Equalizing Noise (TEN) test is used to identify the presence of DRs

- A DR at a CF is indicated by a masked threshold $\geq 10 \mathrm{~dB}$ above the absolute threshold and $10 \mathrm{~dB}$ above the nominal noise level (Moore 2000)

- Functional hearing outcomes of CI recipients who fulfil extended criteria due to DRs is unknown
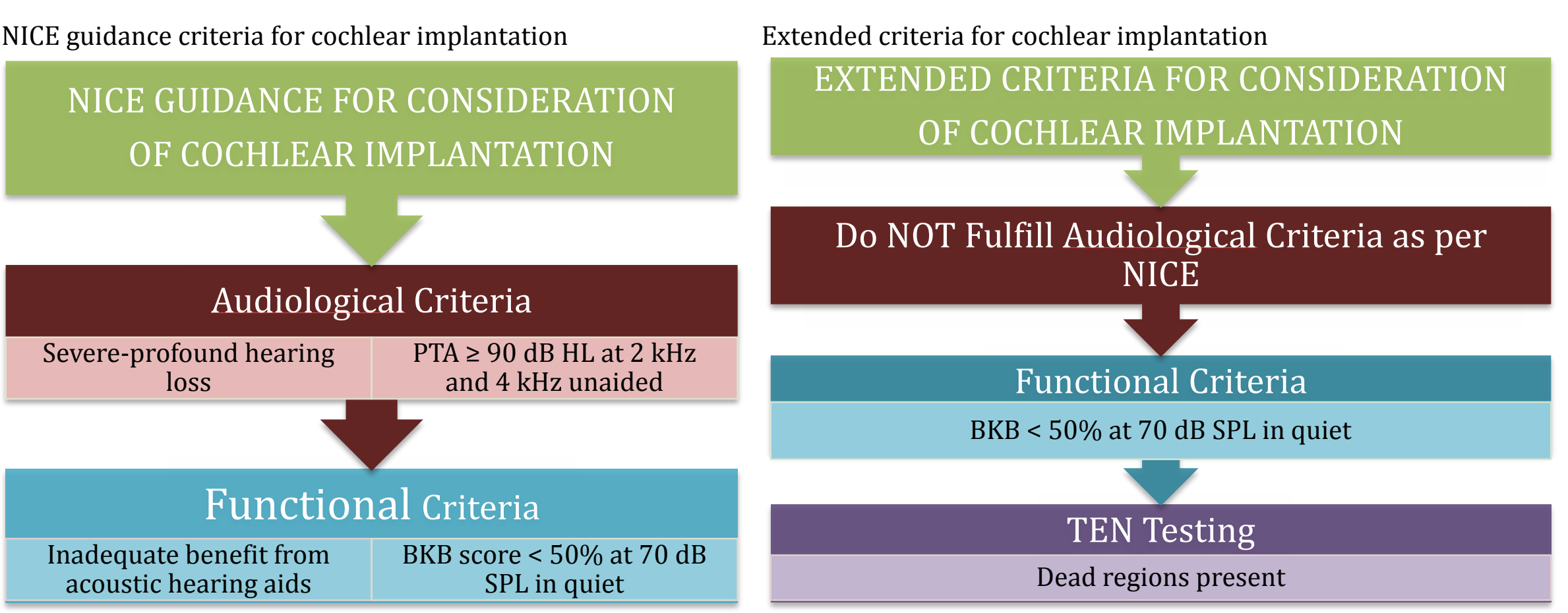

Methods

- Retrospective chart review

- All patients receiving CI between Dec 2014 and Oct 2017 in a UK regional implant centre were included

- Patients without pre- and post-op BKB scores excluded

- Patients who fulfilled extended CI criteria with preoperative cochlear DRs were identified using Interacoustics Affinity 2.0 audiometer

- Comparison with control patients who fulfilled traditional CI criteria matched by preoperative BKB

\section{Results: DR Group}

- Total of 47 patients received CIs due to extended criteria

- Mean age at implantation 56 years

- Mean duration of deafness 12 years

- Median preop BKB 23\%

- Median postop BKB 85\%

- 45/47 (95.7\%) had improved BKB postoperatively

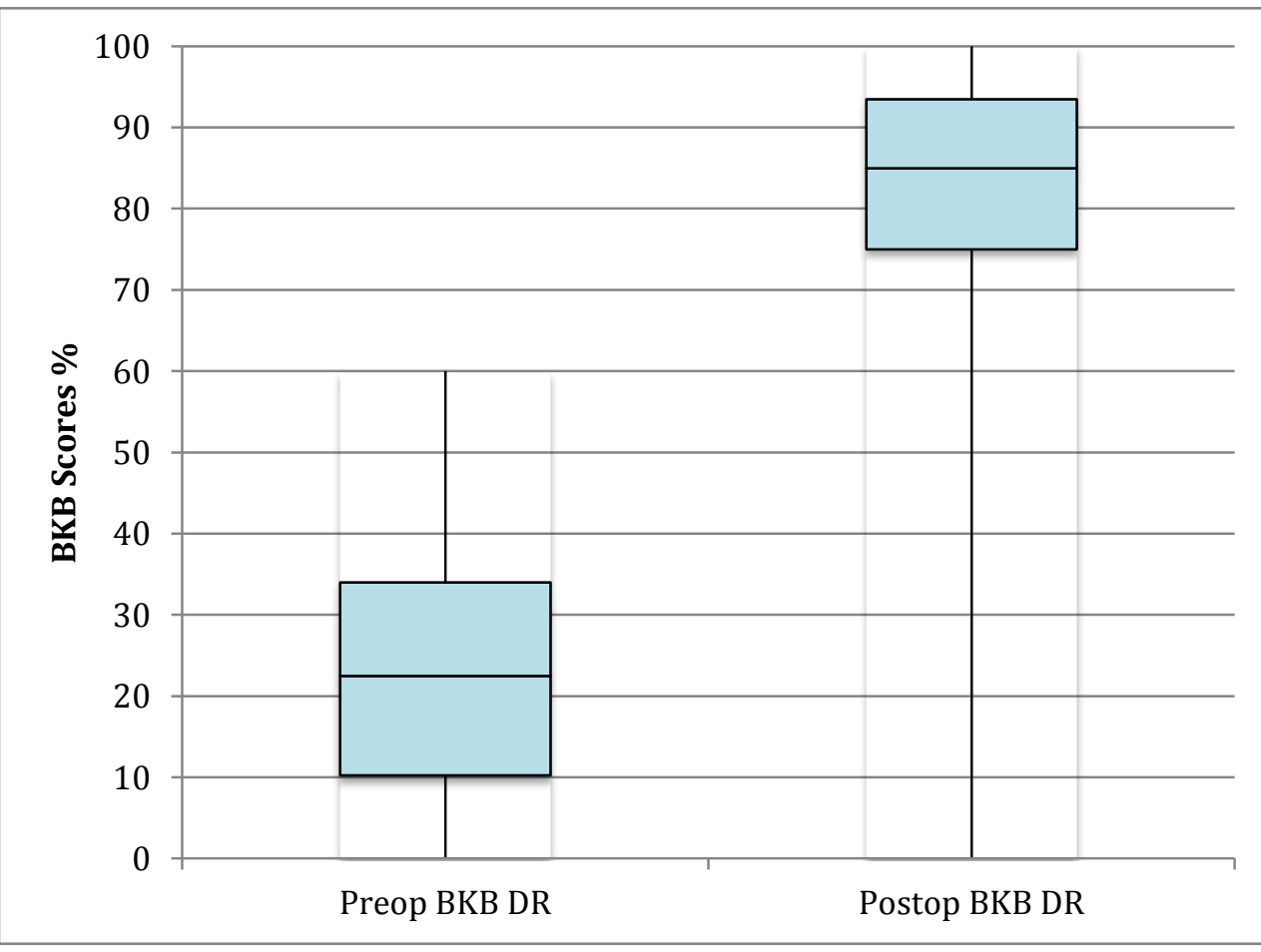

Results: DR Group vs Matched Controls

- Total of 34 comparisons between DR group and matched controls

- Mean age at implantation 60.7 vs 57.4 years $(p=0.28)$

- Median preop BKB 19\%

- Median postop BKB 86.5 vs $82.5 \%$ $(\mathrm{p}=0.31)$
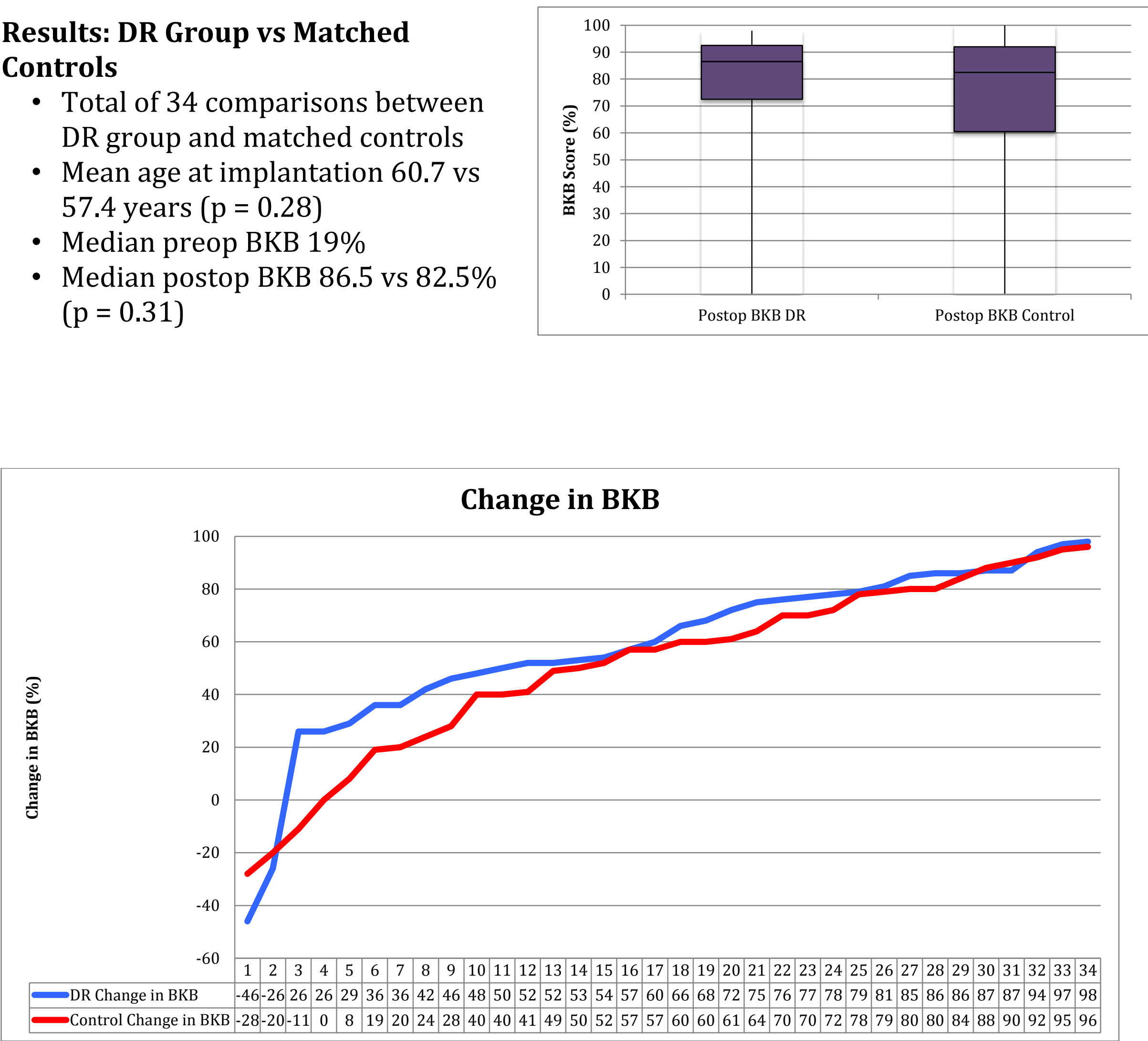

\section{Discussion}

- Largest series to date looking at hearing outcomes of CI recipients who fulfilled extended criteria based on cochlear DRs

- Only series comparing DR patients to matched controls

- Moualed 2018: 6 CI patients with DRs, mean BKB 25\% preop to 80\% postop

- Limitations:

- Retrospective study, small numbers

- Insufficient data to exclude duration of deafness as potential confounder

- Patients with auditory neuropathy or central lesions have high thresholds on TEN test

- Good outcomes with CI suggests pathology in IHCs

Conclusions

- Patients who fulfill extended criteria for CI based on presence of cochlear DRs have comparable functional outcomes to patients who fulfill traditional NICE criteria

- For patients functionally poor hearing with identified cochlear DRs who have inadequate benefit from HAs, cochlear implantation likely to be a better option

- TEN testing may identify potential candidates for CI who fail to meet traditional audiological criteria and should be part of standard work-up for CI

References

- Moore BCJ, Huss M, Vickers DA, Glasberg BR, Alcantara JI. A test for the diagnosis of dead regions in the cochlea. British Journal of Audiology (2000); 34(4):205-224

Moualed D, Humphries J, Ramsden JD. Cochlear dead regions: using the threshold equalising noise (TEN) test to improve the assessment of potential cochlear implant candidates- the Oxford experience. Clinical Otolaryngology (2018) 